\title{
Do radionuclide and echocardiographic techniques give a universal cut off value for left ventricular ejection fraction that can be used to select patients for treatment with ACE inhibitors after myocardial infarction?
}

\author{
S G Ray, M J Metcalfe, K G Oldroyd, M Pye, W Martin, J Christie, H J Dargie, \\ $S M$ Cobbe
}

\begin{abstract}
Objective-To determine whether echocardiography and radionuclide angiography give comparable results when the left ventricular ejection fraction is measured early after myocardial infarction and thus whether, irrespective of the method used, a single value for the ejection fraction could be used as a guide for starting treatment with an angiotensin converting enzyme inhibitor.

Design-Prospective comparison of measurement of left ventricular ejection fraction by echocardiography and radionuclide angiography.

Setting-Coronary care units of two university teaching hospitals in Glasgow.

Patients-99 patients studied within 36 hours of acute myocardial infarction.

Outcome measures-Left ventricular ejection fraction assessed by echocardiography and radionuclide angiography. Results-70 (77\%) of the 99 patients had ejection fraction measured by both echocardiographic and radionuclide techniques, 30 in centre 1 and 40 in centre 2 . In centre 1 the mean difference (SD) in ejection fraction (radionuclide angiography - echocardiography) was $-8(10 \%) ; 95 \%$ CI -12 to $-4 \%$. In centre 2 the mean difference was -14 (11\%); $95 \%$ CI -17 to $-11 \%$. If patients had been treated with an ACE inhibitor on the basis of a radionuclide ejection fraction of $<40 \%$ then $93 \%$ in centre 1 ( 28 of 30 ) and $98 \%$ in centre 2 (39 of 40) would have been treated. This compares with $63 \%$ (19 of 30 ) and $50 \%$ (20 of 40 ), respectively if echocardiography had been used as a guide.

Conclusion-Measurement of ejection fraction is highly dependent on the method used and it is therefore impossible to quote a universally applicable figure for left ventricular ejection fraction below which an ACE inhibitor should be used after myocardial infarction.
\end{abstract}

(Br Heart f 1995;73:466-469)
Keywords: left ventricular ejection fraction; echocardiography; radionuclide angiography; ACE inhibitors.

In the recently published AIRE trial patients with signs of heart failure after myocardial infarction survived longer when they were treated with the ACE inhibitor ramipril, even if the signs of heart failure were only transient. ${ }^{1}$ This is a very important trial because it provides simple clinical guidelines for the treatment of a high risk group. If clinical signs of heart failure were used exclusively to select patients for treatment with an ACE inhibitor, however, many patients likely to benefit would be excluded. ${ }^{2}$ The Survival and Ventricular Enlargement (SAVE) trial showed that patients with left ventricular dysfunction but no symptoms also survive longer when treated with an ACE inhibitor. ${ }^{3}$ Widely applicable measures to identify this latter group have yet to be defined but the method most commonly used is the estimation of left ventricular ejection fraction. In the SAVE trial an ejection fraction of $<40 \%$ was used as the threshold for randomisation and this is now being adopted in clinical practice. One potential difficulty with this approach is the variation in ejection fraction when measured by different methods and in different centres. In the SAVE trial ejection fraction was measured by a radionuclide technique. ${ }^{4}$ In the United Kingdom only about a third of general hospitals have on site nuclear medicine facilities and most must therefore measure ejection fraction by echocardiography, the only other widely available technique. We set out to determine whether echocardiography and nuclear imaging give comparable results when used to assess left ventricular ejection fraction early after myocardial infarction and thus, by implication, whether a single widely applicable figure could be used to guide treatment with ACE inhibitors.

\section{Patients}

We studied 99 haemodynamically stable patients between 6 and 24 hours after myocardial infarction. Principal entry criteria 
were: (a) ST elevation of at least $3 \mathrm{~mm}$ in two precordial leads V1-V4 and/or $2 \mathrm{~mm}$ in two frontal plane leads or in V5-V6 or any ST elevation in two frontal plane leads with ST depression compatible with posterior wall infarction of at least $3 \mathrm{~mm}$ in two precordial leads. (b) Norris Index $>3 \cdot 5.5^{5}$ (c) Systolic blood pressure $\geqslant 95 \mathrm{~mm} \mathrm{Hg}$.

These entry criteria were designed to exclude patients with small infarcts and those with haemodynamic instability or cardiogenic shock. The characteristics of the population studied and their short and medium term outcome have been described in detail elsewhere. ${ }^{67}$ Left ventricular function was assessed as soon as possible after admission by both cross sectional echocardiography and radionuclide ventriculography. The examinations were performed in no set order and in most patients both were completed within 4 hours of each other. In a few cases radionuclide ventriculography was delayed for a maximum of 12 hours because the gamma camera was not immediately available. All studies were completed about 36 hours after the onset of symptoms.

\section{ECHOCARDIOGRAPHIC MEASUREMENT OF EJECTION FRACTION}

There were differences in the echocardiographic methods used to measure ejection fraction at both centres. In the first centre long axis parasternal and apical two and four chamber images of the heart were obtained with a Hewlett Packard 770200 imaging system. We measured left ventricular dimensions in short and long axes with the internal callipers of the machine and obtained the left ventricular ejection fraction using a simple scientific calculator by entering these figures into the equation derived by Quinones et al. ${ }^{8}$ In the second centre echocardiographic left ventricular ejection fraction was measured by a 10 segment Simpson's rule method. ${ }^{9}$ This required acquisition of apical four chamber and two chamber views and calculations of ejection fraction were made on line using the internal software package of an ATL Ultramark 8 imaging system. The methods of calculation were constrained by the different software packages available with the imaging equipment used and were chosen to allow rapid estimation of ejection fraction without the need for time consuming off-line analysis or expensive additional software. All echocardiograms were obtained by one of three experienced operators and the mean of three cardiac cycles was taken. The standard deviation of repeated measures (10 duplicates for each operator incorporating both techniques) was $3 \cdot 4 \%$.

\section{RADIONUCLIDE VENTRICULOGRAPHY}

Radionuclide ventriculography was performed after conventional in vivo labelling with $800 \mathrm{MBq}$ of technetium-99m pertechnate and the subsequent acquisitions were performed in the standard left anterior oblique projection. ${ }^{10}$ This projection avoids any significant overlap with the right ventricle and the left atrium. The study was then collected in frame mode by a Siemens portable gamma camera in a $64 \times 64$ matrix, with a $\times 2$ zoom facility (total collection 4 million counts). A low energy, general purpose, collimator was used, optimised to 140 (10) $\mathrm{kV}$. The study was formatted using a $50 \mathrm{~ms}$ time window and phase, amplitude, and stroke volume images were calculated using a standard LINK computer and MAPS 5000 software.

The left ventricular ejection fraction was calculated by drawing a left ventricular region of interest around an end diastolic frame of the left ventricle using the amplitude and phase images for guidance. A standard background region of interest was drawn and the ejection fraction was calculated from the change in counts between end diastole and end systole correcting for background. The method of acquisition and analysis was identical in both centres. The standard deviation of repeated measurements was $1.9 \%$.

\section{Results}

Left ventricular function was assessed by one or other method in 91 of the 99 patients recruited to the study. The remaining 8 patients died or were withdrawn before imaging could be performed or were in atrial fibrillation, which effectively excludes accurate measurement of ejection fraction. Echocardiographic ejection fraction was measurable in $77(85 \%)$, the remaining patients having images of insufficient quality for analysis. Eighty four patients (92\%) underwent radionuclide angiography and all studies were of analysable quality. Seven patients ( $8 \%$ ) could not be examined because the gamma camera was not available. Seventy patients $(77 \%)$ had ejection fraction assessed by both echocardiography and radionuclide ventriculography. Of these 30 were studied in centre 1 and 40 in centre 2 . In centre 1 the mean radionuclide ejection fraction (24 $(10) \%)$ was substantially lower than the echocardiographic ejection fraction measured by the method of Quinones (32 (12)\%). The mean difference (RNVG - echo) was -8

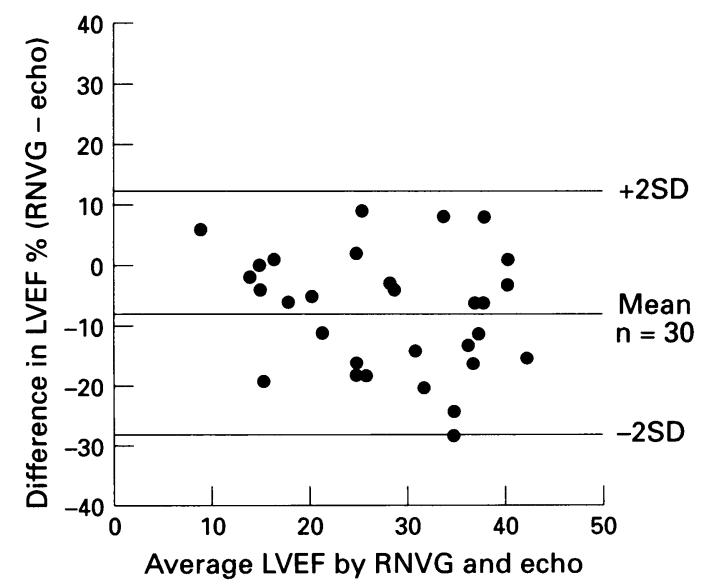

Figure 1 Relation between left ventricular ejection fraction measured by echocardiography (echo) and radionuclide angiography (RNVG) in centre 1. 
Figure 2 Relation between left ventricular ejection fraction measured by echocardiography (echo) and radionuclide angiography (RNVG) in centre 2 .

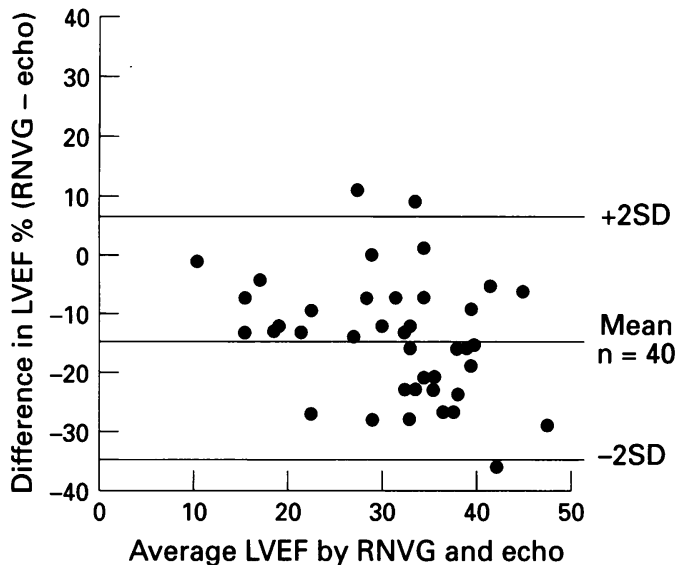

(10) $\%$; $95 \%$ CI -12 to -4 , limits of agreement $-28 \%$ to $+12 \%$ (fig 1 ). In centre 2 the differences were even more pronounced. Mean ejection fraction by radionuclide imaging was 24 (9)\% and by echocardiography using Simpsons Rule it was 38 (12)\%. The mean difference (RNVG - echo) was - 14 (11) $\%$; $95 \%$ CI -17 to -11 and the limits of agreement extremely wide at $-35 \%$ to $+8 \%$ (fig 2).

If patients had been treated with an ACE inhibitor on the basis of a radionuclide ejection fraction of $<40 \%$ then $93 \%$ of patients in centre 1 (28 of 30 ) and $98 \%$ in centre 2 (39 of 40) would have been treated. This compares with $63 \%$ (19 of 30 ) and $50 \%$ (20 of 40) respectively if echocardiographic measurements had been used.

\section{Discussion}

It is evident from these results that it is impossible to quote a universally applicable figure for left ventricular ejection fraction below which an ACE inhibitor should be prescribed after myocardial infarction. Neither of the echocardiographic methods used in this study showed a workable agreement with radionuclide imaging. Previous studies obtained closer agreement between echocardiographic and nuclear techniques but generally avoided patients with previous myocardial infarction. ${ }^{911}$ Albin and Rahko compared nuclear techniques and various echocardiographic methods in patients after infarction. ${ }^{12}$ With methods incorporating short axis views the discrepancy (nuclear echo) was about $-7 \%$, similar to the difference seen with the method of Quinones et al in our study. ${ }^{8}$ The closest correlation was achieved with a 30 segment Simpson's rule apical biplane method-when the mean difference in ejection fraction was only $-2 \%$. In our series the difference between the radionuclide ejection fraction and that measured by a 10 segment Simpson's apical biplane method was $-14 \%$. We cannot be certain of the reason for the considerable discrepancy in these results. Simpson's rule analysis increases in accuracy with the number of segments analysed, but it is inconceivable that an increase from 10 to 30 segments could explain such a large variation. Although both studies in the paper by Albin and Rahko were performed within 48 hours of each other it is not clear whether infarct patients were studied in the subacute or chronic phase. ${ }^{12}$ Estimations of ejection fraction are more likely to be reproducible in the chronic phase of myocardial infarction and this might contribute to the closer correlation they observed. Albin and Rahko used sophisticated off-line analysis of the echocardiogram, which is not available or practical in most hospitals. ${ }^{12}$ Our aim was to use techniques that did not require additional sophisticated software and that could be used in routine clinical practice in a district general hospital.

We were unable to compare directly the two echocardiographic methods because the ultrasound systems used were not compatible. The mean ejection fractions measured by the same nuclear technique in the two centres were identical (24\%). Despite this there was a discrepancy of $6 \%$ in the mean ejection fractions measured by echocardiography. This implies that there is likely to be a systematic difference in the value of ejection fraction given by the two echocardiographic methods. Although radionuclide measurements are probably more reproducible than echocardiographic ones and less subject to poor image quality there is considerable variation between centres in what is regarded as a normal ejection fraction. A recent survey by the British Nuclear Cardiology Group ${ }^{10}$ found that the figure quoted for the lower limit of the normal range of left ventricular ejection fraction by radionuclide angiography ranged from $35 \%$ to $75 \%$ (median $50 \%$ ) in different centres. The important practical issue is not whether one method is intrinsically better than the other but that echocardiographic and radionuclide angiographic methods suitable for routine use do not necessarily provide comparable results. Moreover, neither method is sufficiently independent of interhospital variation in technique to allow the universal adoption of a single figure as a cut off for the use of ACE inhibition after infarction. The use of ejection fraction to guide treatment is only meaningful when applied in the context of a locally derived normal range specific to the method of measurement.

It is possible that other screening methods might supersede assessment of ejection fraction in the selection of patients likely to benefit from an ACE inhibitor. One such possibility is measurement of brain natriuretic peptide, which is raised in plasma from patients with asymptomatic left ventricular dysfunction. ${ }^{13}$ There is also some evidence that late potentials on a signal averaged echocardiogram in the early post-infarction period might predict which patients are at risk of ventricular dilatation and thus likely to benefit for ACE inhibitor treatment. ${ }^{14}$ Currently, however, measurement of ejection fraction is an important part of the screening process in many patients.

In conclusion, the estimation of ejection fraction must be interpreted within the context of the method used and with reference to 
a locally defined normal range. A single figure cannot be used as a universal determinant for the use of an ACE inhibitor after myocardial infarction.

1 The Acute Infarction Rampipril Efficacy (AIRE) Investigators. Effect of ramipril on mortality and morbidity of survivors of acute myocardial infarction with evidence of heart failure. Lancet 1993;342:821-7.

2 McMurray J. ACE inhibitors after myocardial infarction. Lancet 1993;342:1418.

3 Pfeffer MA, Braunwald E, Moye L, et al. Effect of captopril on mortality and morbidity in patients with left venpril on mortality and morbidity in patients with left ven-
tricular dysfunction after myocardial infarction. $N$ Engl tricular dysfunction after

4 Moye L, Pfeffer MA, Braunwald E. Rationale, design, and baseline characteristics of the survival and ventricular ;68:79D-9D.

5 Norris RM, Brandt PWT, Caughey DE, Lee AJ, Scott PJ. A new coronary prognostic index. Lancet 1969 ;i: 2

6 Oldroyd KG, Pye MP, Ray SG, et al. Effects of early captopril administration on infarct expansion, left ventricular remodelling, and exercise capacity after acute myocardial infarction. Am $\mathcal{F}$ Cardiol 1991;68:713-8.

7 Ray SG, Pye MP, Oldroyd KG, et al. Early treatment with captopril after acute myocardial infarction. Br Heart $\mathcal{F}$ 1993;69:215-22
8 Quninones MA, Waggoner AD, Reduto LA, et al. A new, simplified and accurate method for determining ejection fraction with two dimensional echocardiography Circulation 1981;64:744-51

9 Starling MR, Crawford MH, Sorensen SG, Levi B, Richards KL, O'Rourke RA. Comparative accuracy of apical biplane cross-sectional echocardiography and gated radionuclide angiography for estimating left ventricular size and performance. Circulation 1981;63. 1075-83.

10 Underwood SR, Gibson C, Tweddel AC, Flint J. A survey of nuclear cardiological practice in Great Britain. $B r$ Heart f 1992;67:273-7.

11 Folland ED, Parisi AF, Moynihan PF, Jones DR, Feldman CL, Tow DE. Assessment of left ventricular ejection fraction and volumes by real time two dimensional echocardiography. A comparison of cineangiographic and radionuclide techniques. Circulation 1979; 6raphic and

12 Albin G, Rahko PS. Comparison of echocardiographic quantification of left ventricular ejection fraction to radionuclide angiography in patients with regional wal motion abnormalities. Am f Cardiol 1990;65:1031-2.

13 Motwani JG, Mc Alpine HM, Kennedy N, Struthers AD Plasma BNP as an indicator for ACE inhibition after myocardial infarction. Lancet 1993;341:1109-13.

14 Zaman AG, Morris JL, Smyllie JH, Cowan JC. Late potentials and ventricular enlargement after myocardia infarction. Circulation 1993;88:905-14. 